\title{
Robust BELBIC-Extension for Trajectory Tracking Control
}

\author{
Sophie Klecker, Bassem Hichri and Peter Plapper \\ The Faculty of Science, Technology and Communication, University of Luxembourg, Luxembourg L-1359, Luxembourg
}

\begin{abstract}
In real-life trajectory tracking applications of robotic manipulators uncertain robot dynamics, external disturbances and switching constraints which cannot be accommodated for by a conventional controller affect the system performance. We suggested an additional control element combining sliding mode and bio-mimetic, neurologically-inspired BELBIC (brain emotional learning-based intelligent control). The former is invariant to internal and external uncertainties and guarantees robust behavior. The latter is based on an interplay of inputs relating to environmental information through error-signals of position and sliding surfaces and of emotional signals regulating the learning rate and adapting the future behaviour based on prior experiences and with the goal to maximize a reward function. We proofed the stability and the performance of the suggested control scheme through Lyapunov theory and numerical simulations, respectively.
\end{abstract}

Key words: Intelligent control, sliding mode control, biomimetics, robotic manipulator, switching constraints, trajectory tracking, freeform following.

\section{Introduction}

Conventional controllers perform well in robotic trajectory tracking applications for the idealized case only. This idealized case is characterized, first, by a lack of system-inherent uncertainties, i.e., by perfectly known robot dynamics and kinematics, second, by a lack of external disturbances and third, by an a priori well-known smooth continuous trajectory with fixed constraints.

In real-life applications, however, contrary to under idealized circumstances, external as well as internal uncertainties undermine the performance. The system being implemented in and interacting with its environment, external disturbances negatively affect the stable system behaviour. Additionally, uncertain robot dynamics and parameters deteriorate the overall efficiency.

Sliding mode control, is considered a prominent robust control strategy as it is invariant to both internal

Corresponding author: Sophie Klecker, doctoral candidate, research fields: robotics and manufacturing engineering. and external disturbances. A high-speed switching control law guiding the system's state trajectory onto a user-defined sliding surface in the state space and keeping it there for subsequent times, allows the control of highly nonlinear systems in the presence of uncertainties. The major disadvantages of the variable structure control method are first, chattering, i.e., the occurrence of undesired vibrations and second, the lack of a priori knowledge about function bounds. Extensions with elements of intelligent control as neural networks by Van Cuong et al. [1] or fuzzy logic by Roopaei et al. [2], Li et al. [3], Li et al. [4] were introduced to overcome these drawbacks.

A change in constraints between the end-effector of the robot and the environment results in a switched nonlinear system. In contrast to fixed constraints, switching constraints seriously interfere with the overall system's stability as shown by Liberzon [5]. Pagilla et al. [6] suggested an event-based control switching strategy for a surface finishing process. The different process phases are identified and tackled with a respectively adapted control algorithm. A strategy combining an intelligent adaptive neural controller 
with a robust compensator is proposed by Yu et al. [7]. While the robust compensation control law accommodates internal and external disturbances, the switching neural controller approximates any unknown function. Jasim et al. [8] addressed the problem of a robotic manipulator with continuously switching constraints with an adaptive sliding mode controller.

The control of a nonlinear system with switching constraints in the presence of uncertainties finally requires the combination of a robust control strategy and an intelligent control element.

The skills that we want to add to the controller through the addition of an intelligent control element are natural human skills. The choice for a biomimetic controller seems therefore most appropriate. Indeed, biomimetics aims for human-made solutions mimicking biological solutions, thereby taking advantage of the strength of natural evolution. Recently bio-inspired engineering has celebrated resounding successes due to its innovative, relatively simple and intuitive solutions to highly complex problems, particularly in robotic trajectory tracking. Tramper et al. [9] studied the human's combination of anticipation and ongoing feedback control for contour following tasks. Huang et al. [10] investigated how a contact force is adapted if it suddenly deviates from the expected or desired force. In Yin et al. [11] the hierarchical architecture of the human tactility was imitated to make a robot track an unknown surface.

Lucas et al. [12] developed a controller based on the emotional learning behaviour of the mammalian brain: BELBIC (brain emotional learning based intelligent control). The source of inspiration for this controller was the emotional learning behaviour of the Amygdala-Orbitofrontal system of the human brain and more precisely, Moren and Balkenius' computational model of the abstracted human limbic system comprised of the amygdala and the orbitofrontal cortex [13, 14]. The core idea is to attach emotions to inputs and their corresponding outputs. A reward function, i.e., a positive emotion is attempted to be maximized through adaptation of current or future behaviour based on prior experiences. This is the innate behaviour which is observed throughout mammalians. The key advantages of this control strategy are improved learning capabilities, fast training, robustness, reduced computational burden and an intuitive algorithm. Lately, many engineering disciplines ranging from power systems by Aghaee et al. [15] to induction motors by Daryabeigi et al. [16] have adapted this neurologically inspired control strategy. The work by Yi [17] showed that a combination of sliding mode and brain limbic system control outperforms a conventional as well as a fuzzy sliding mode controller for trajectory tracking applications.

This paper combines elements of the work by Jasim et al. [8] on adaptive sliding mode control of a switched nonlinear system and of the work by Yi [17] on a strategy combining sliding mode with brain limbic system control. This combination allows a stable trajectory tracking performance, even in complex, uncertain environments like the following of discontinuous freeform surfaces, a topic which is relevant i.e., in contact-based manufacturing processes like freeform grinding. BELBIC compensates uncertain switching constraints while sliding mode control accommodates internal and external uncertainties. The suggested control scheme is applied to the following of discontinuous freeform paths.

The rest of the paper is structured in the following manner: Section 2 describes the problem and is followed by the design of the BELBIC-SMC-controller adapted for the following of discontinuous freeform surfaces by robotic manipulators under the presence of uncertainties. Section 3 also contains the proof of stability by Lyapunov-theorem. In Section 4, the suggested control scheme is validated through simulation of a 2-link robot in the Matlab/Simulink environment. Section 5 consists of concluding remarks. 


\section{The Robotic Manipulator}

The dynamics of the considered n-link robotic manipulator with switching constraints and disturbances can be expressed in Lagrange form.

$$
M(q) \ddot{q}+C(q, \dot{q})+G(q)=u+Q_{i}+\tau
$$

where, $q, \dot{q}, \ddot{q} \in R^{n}$ are the link position, velocity and acceleration, $M(q) \in R^{n x n}$ is the inertia matrix, $C(q, \dot{q}) \in R^{n}$ is the centripetal/Coriolis vector, $G(q) \in R^{n}$ is the vector of gravitational torque, $\tau \in R^{n}$ is the bounded external disturbance and $u \in R^{n}$ is the vector of applied input torque. $Q_{i} \in R^{n x 1}$ is the global constraint force, $Q_{i}=$ $J^{T}(q) D_{i}^{T}(\alpha) \lambda$ where $J(q) \in R^{n x 6}$ is the manipulator's Jacobian, $\lambda \in R^{m}$ is the vector of Lagrange multipliers and $D_{i}(\alpha)=\frac{\delta \phi_{i}(\alpha)}{\delta \alpha}$ is the gradient of the task space constraints with $\emptyset_{i}(\alpha) \in$ $R^{6}$ the $\mathrm{i}^{\text {th }}$ kinematic constraint due to the system's environment. Here $\alpha \in R^{6}$ stands for the Cartesian pose, i.e., Cartesian position and orientation whereas $i=1,2, \ldots m$ denotes the index of constraints for the case of multiple switching constraints with $m$ being the total number of constraints. As an illustration: When $i=3$, the third constraint is active while the other constraints are inactive.

\section{The Controller}

The topic of this paragraph is the control for path following of a class of robotic manipulators in the presence of switching constraints and disturbances. Robust behaviour even in the case of external disturbances is guaranteed through the use of a sliding mode controller. To overcome the drawbacks of the sliding mode controller and to adapt to any given output signal, a bio-inspired BELBIC-control term is added. The suggested robust control law structure comprises two parts: $u_{n}$, the nominal term, similar to a conventional controller and $u_{b}$ a compensation-term for unpredictable uncertainties and disturbances. $u_{n}$ and $u_{b}$ are summed up to form $u$ which is the total input vector.

$$
u=u_{n}+u_{b}
$$

With $u_{n}, u_{b}, u \in R^{n}$.

Fig. 1 illustrates the suggested control scheme by means of a block diagram.

The conventional, equivalent control input is based on weighted position error-signals $q_{\text {error }}, q_{\text {error }}, \ddot{q_{r}} \in$ $R^{n}$

$$
\begin{gathered}
q_{\text {error }}=q-q_{d} \\
q_{\text {error }}=\dot{q}-\dot{q}_{d} \\
\ddot{q_{r}}=\ddot{q_{d}}-N_{1} q_{\text {error }}
\end{gathered}
$$

where, $q_{d}, \dot{q}_{d}, \ddot{q}_{d}, q, \dot{q}, \ddot{q} \in R^{n}$ express the desired or current link position, velocity and acceleration, respectively and $N_{1}=n_{1} I_{n}, N_{2}=n_{2} I_{n} \in R^{n x n}$ are independent, constant square diagonal gain matrices, $n_{1}, n_{2}$ are constant factors and $I_{n}$ is an $\mathrm{n} \times \mathrm{n}$ identity matrix.

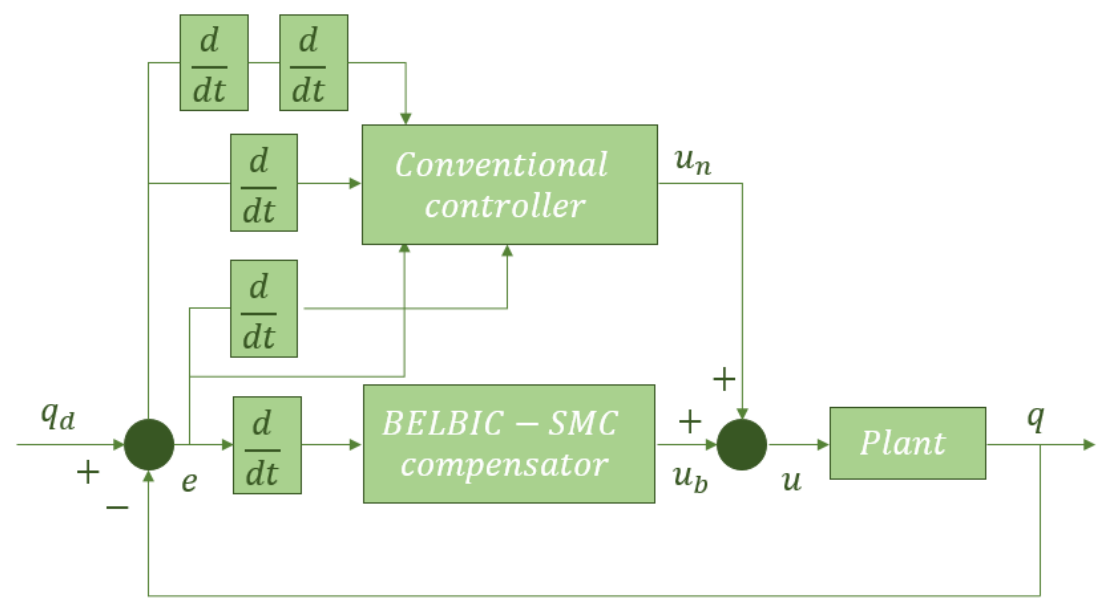

Fig. 1 Block diagram of the developed control scheme. 
The equivalent term of the control input is expressed as follows

$$
u_{n}=M(q)\left(\ddot{q}_{r}-N_{2} q_{\text {error }}\right)+C(q, \dot{q}) \dot{q}+G(q)
$$

$u_{b}$, the second control input term combines elements of a robust sliding mode controller and artificial intelligence, a BELBIC controller.

Sliding mode control is prominently chosen for controlling systems with strong nonlinearities as well as parametric and modelling uncertainties. A high-speed switching control law is used to first, guide the system's state trajectory onto a user-defined switching or sliding surface in the state space and to, second keep it on this sliding surface for subsequent times. The sliding surface $s \in R^{n}$ is defined as a weighted sum of position and velocity errors.

$$
s=q_{\text {error }}+S_{1} q_{\text {error }}
$$

where, $S_{1}=s_{1} I_{n} \in R^{n x n}$ is an independent, constant, positive-definite square diagonal gain matrix satisfying $S_{1}<N_{1}$ and $s_{1}$ is a constant factor.

A side effect of variable structure control is chattering, undesired oscillations resulting from imperfect control switchings. In theory, an infinitely fast switching allows to keep the system's state trajectory on the smooth sliding surface. In practice, however, switching is restricted to a finite frequency and a resulting oscillation around the switching surface is observed. A well-known remedy to this phenomenon is a saturation function as replacement for the signum function of the sliding surface in the control output term by Liu et al. [18], Slotine et al. [19].

$$
\text { if } \begin{aligned}
\left|s_{i}\right|>\Delta_{i}: \text { sats }_{i} & =\operatorname{sign}\left(s_{i}\right) \text { else: } \text { sats }_{i}=\frac{1}{\Delta_{i}} s_{i} \\
i & =1,2, \ldots, n
\end{aligned}
$$

where, $\Delta_{1}=\Delta_{2}=\cdots=\Delta_{i}=\Delta_{n}$ is the boundary layer defining the region in which switching control is to be applied.

Although sliding mode control has proven good robustness and tracking performance for a vast range of use cases, two major drawbacks limit its use for specific applications. First, chattering is only unsatisfactorily solved by the introduction of a saturation function. Second, conventional sliding mode control requires the knowledge of the function's constraints bounds which is hardly possible to acquire.

To overcome the drawbacks of sliding mode control, it is combined with elements from intelligent control. Improved learning capabilities including fast training, robustness, reduced computational burden and an intuitive algorithm are key reasons for opting for neurologically-inspired BELBIC. BELBIC was introduced by Lucas et al. [12] following the work of Morén and Balkenius [13, 14] on a computational model of the emotional learning behaviour of the mammalian brain. In the network like structure of the amygdalo-orbitofrontal system, the amygdala, the actuator, maps sensory stimuli to their linked emotional responses while the orbitofrontal cortex, the preventer, inhibits connections as a response to changing aspirations or environment. The emotional stimulus-response's capacity to assist in making fast decisions is exploited in the framework of two-process learning and signal processing resulting in an action-generator (one output) based on sensory inputs and emotional cues (multiple inputs). The suggested control law is based on an interplay of inputs relating to environmental information through error-signals of position and sliding surfaces and of emotions regulating the learning rate and adapting the future behaviour based on what has been learned.

Fig. 2 summarizes the network like structure of the amygdalo-orbitofrontal system as considered in this work by Aghaee et al. [15], Daryabeigi et al. [16], Yi [17].

Eqs. (9)-(16) in the following paragraph are to be considered as scalar notation for the individual links of the considered n-link robotic manipulator. Solely for legibility reasons an indexed notation as presented in Eq. (8) is omitted.

The sensory input can be considered as an input-perception of the environment.

$$
s i=\sigma s
$$




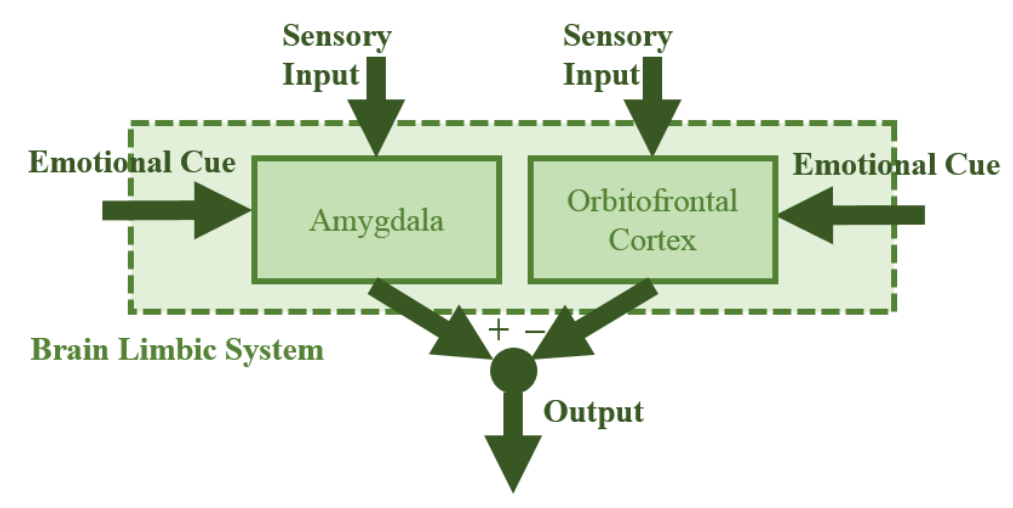

Fig. 2 BELBIC-structure.

si $\in R^{n}$ is modelled as a weighted sliding surface term where $\sigma$ is an arbitrary constant factor.

Complementing the objective sensory input, a reinforcing subjective emotional cue function is introduced. The goal of this reward function being to ever increase the reward, an emotional cue function $e c \in R^{n}$ whose maximum values are situated in the target regions is suggested. Based on the type of emotion that is envisaged for the specific application, it makes usage of error signals as well as weighted sensory input and control input terms.

$$
e c=\left(\varepsilon_{1}-\varepsilon_{2}\right)\left(s i-\epsilon u_{b}\right)
$$

where, $\epsilon$ is a constant arbitrary factor.

$\varepsilon_{1}, \varepsilon_{2} \in R^{1}$ express the Cartesian distance between position- and velocity-errors of successive manipulator links. As an illustration: for 2 links

$$
\begin{gathered}
\varepsilon_{1}=\sqrt{q_{\text {error }_{1}}{ }^{2}+q_{\text {error }_{2}}^{2}} \\
\varepsilon_{2}=\sqrt{q_{\text {error }_{1}}^{2}+q_{\text {error }}{ }^{2}}
\end{gathered}
$$

The output of the amygdala $a \in R^{n}$, the system's actuator mapping sensory stimuli to their related emotional responses is expressed in terms of Eq. (12).

$$
a=\operatorname{si} g_{a}
$$

where, $g_{a}$ is a connection weight which is adjusted according to the gain function $\Delta g_{a} \in R^{1}$ of the learning process. It expresses proportionality to the difference between reward function, i.e., emotional cue signal and amygdala-output.

$$
\Delta g_{a}=\gamma(\operatorname{simax}(0, e c-a))
$$

where, $\gamma \in R^{1}$ is the associated constant learning rate.
The sensory inputs to the amygdala are multiplied by the introduced weight function in a monotonic manner, i.e., the weights cannot decrease. This mimics the natural learning behaviour where all learned emotional responses are permanent and where the inhibition of no longer appropriate connections is the orbitofrontal cortex' duty.

$$
o c=s i g_{o c}
$$

$o c \in R^{n}$, the output of the orbitofrontal cortex expresses the reaction to changes in environment or aspiration, where $g_{o c}$ is a connection weight adjusted following $\Delta g_{o c} \in R^{1}$ in proportion to the difference between previous output and emotional cue signal.

$$
\Delta g_{o c}=\beta(s i(o-e c))
$$

where, $\beta \in R^{1}$ is the associated constant learning rate and $\beta>\gamma$.

The orbitofrontal learning rule, in contrast to the amygdaloidal rule is not monotonic, which is a requirement for performing the inhibitory tasks.

$$
o=a-o c
$$

The single output $o \in R^{n}$ finally subtracts the inhibitory orbitofrontal outputs from the amygdaloidal outputs.

Combining the advantages of both strategies, a novel robust adaptive learning control term is suggested

$$
u_{b}=-B M(q) \int_{0}^{T} s b d t
$$

where, $B$ is an independent, constant, positive-definite gain vector with length $n$ and $T$ is the total time of the movement. 


$$
\begin{gathered}
s b \in R^{n} \\
s b=\text { sats } * s * o \\
u=M(q)\left(\ddot{q}_{r}-N_{2} q_{\text {error }}\right)+ \\
C(q, \dot{q}) \dot{q}+G(q)-B M(q) \int_{0}^{T} s b d t
\end{gathered}
$$

The final control input, which combines elements of conventional control, sliding mode and intelligent BELBIC can be expressed as precedes.

$$
\begin{gathered}
V=\frac{1}{2} s s>0 \\
\dot{V}=s \dot{S} \leq 0
\end{gathered}
$$

Stability of the suggested control law is proven through a Lyapunov stability analysis. The latter states that stable performance is given when the Lyapunov function is positive while its time derivative is negative.

Rewriting Eq. (21) leads to

$$
\dot{V}=s\left(q_{\text {error }}+S q_{\text {error }}\right)
$$

From Eq. (1) one gets

$$
\ddot{q}=M^{-1}(q)\left[u+Q_{i}+\tau-C(q, \dot{q}) \dot{q}-G(q)\right]
$$

Combining Eqs. (5), (19) and (23) into Eq. (22), rearranging, cancelling out and with $N_{2}=\left(S-N_{1}\right) S$, the following expression is obtained

$$
\dot{V}=s\left(\left(S-N_{1}\right) s-B \int_{0}^{T} s b d t+M^{-1}(q) Q_{i}+M^{-1} \tau\right)
$$

For as far as term $-B \int_{0}^{T} s b d t$ is concerned, sats $* s$ is shown to be always positive,

$$
\begin{aligned}
& \text { if }|s|>\Delta \text { : sats } * s=\operatorname{sign}(s) * s>0 \\
& \text { else: } \text { sats } * s=s \frac{1}{\Delta} s>0
\end{aligned}
$$

$o$ is by definition always positive and $B$ is an independent, constant, positive-definite vector. Therefore the term $-B \int_{0}^{T} s b d t$ is always negative.

The purpose of the compensation-term in the total control input is to compensate for unpredictable environmental changes like switching constraints or disturbances, represented here by the terms $Q_{i}$ and $\tau$. The discussed terms therefore cancel out.
Finally, with the earlier introduced relation $S_{1}<N_{1}$,

$$
\dot{V}=s\left(S_{1}-N_{1}\right) s \leq 0
$$

which, proves the stable performance of the suggested control scheme according to Lyapunov.

\section{Results-Simulation}

The trajectory tracking performance of the developed control strategy is simulated in the Matlab/Simulink-environment. For the simulation a planar robotic manipulator with revolute joints and $n=$ 2 is considered (Fig. 3).

$$
\begin{gathered}
{\left[\begin{array}{ll}
M_{11} & M_{12} \\
M_{21} & M_{22}
\end{array}\right]\left[\begin{array}{l}
{ }_{q_{2}} \\
\ddot{q_{1}}
\end{array}\right]+\left[\begin{array}{ll}
C_{11} & C_{12} \\
C_{21} & C_{22}
\end{array}\right]\left[\begin{array}{l}
\dot{q}_{1} \\
\dot{q_{2}}
\end{array}\right]+\left[\begin{array}{l}
G_{1} \\
G_{2}
\end{array}\right]+\left[\begin{array}{l}
\tau_{1} \\
\tau_{2}
\end{array}\right]} \\
M_{11}=j_{1}+m_{2} l_{1}^{2} \\
M_{12}=m_{2} l_{1} l_{c 2} c_{21} \\
M_{21}=m_{2} l_{1} l_{c 2} c_{21} \\
M_{22}=j_{2}+m_{2} l_{c 2}^{2} \\
C_{11}=0 \\
C_{12}=-m_{2} l_{1} l_{c 2} s_{21} \\
C_{21}=m_{2} l_{1} l_{c 2} s_{21} \\
C_{22}=0 \\
G_{1}=m_{1} g l_{c 1} c_{1}+m_{2} g l_{1} c_{1} \\
G_{2}=-m_{2} l_{c 2} g c_{2}
\end{gathered}
$$

The associated inertia, centripetal/Coriolis and gravity matrix and vector terms are given in Eq. (27) by Shah et al. [20] with $m_{1}, m_{2}$ and $l_{1}, l_{2}$ the masses and lengths of link 1 and link 2 respectively, $g$ the gravitational acceleration $9.8 \mathrm{~m} / \mathrm{s}^{2}$. Further, the masses of both links are assumed to be $1 \mathrm{~kg}$ and both link lengths are assumed to be $1 \mathrm{~m} . l_{c 1}$ and $l_{c 2}$ are the

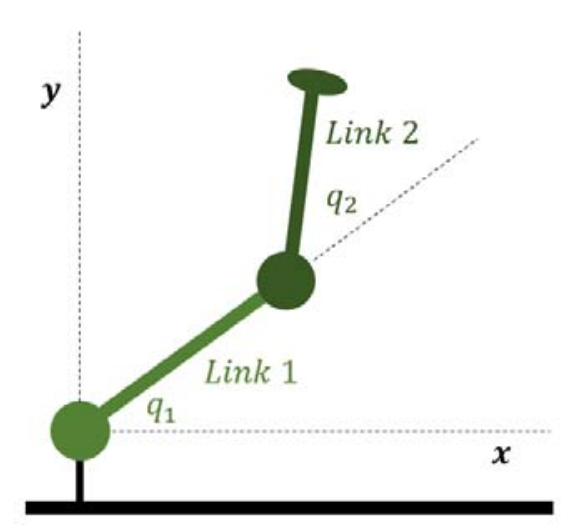

Fig. 3 2-link RR robotic arm. 
distances of the respective link's centre of mass from the source end in m. $j_{1}$ and $j_{2}$ are the moments of inertia of both links about their respective centres of mass in $\mathrm{kg} \cdot \mathrm{m}^{2}$. Also, $s_{1}=\sin \left(q_{1}\right), s_{2}=\sin \left(q_{2}\right)$, $c_{1}=\cos \left(q_{1}\right), \quad c_{2}=\cos \left(q_{2}\right), \quad s_{21}=\sin \left(q_{2}-q_{1}\right)$ and $c_{21}=\cos \left(q_{2}-q_{1}\right)$.

Unpredictable interferences are added to the simulation in the form of switching constraints as well as internal and external disturbances. External, environmental disturbances are added as time-dependent 2-dimensional function. Internal, system-inherent uncertainties are added in the form of $10 \%$ deviations from the expected values in the inertia, centripetal/Coriolis matrices and gravitational torque vector. Switching constraints are implemented in the form of a desired trajectory switching between different curved and straight segments. The defined trajectory is depicted in the x-y-plane in Fig. 4.

The chosen test path includes a concave trajectory interrupted by a switch to another concave trajectory which comprises a small unevenness mimicking imperfect surface conditions in real world applications.

For the simulation the following numerical values are chosen for the parameters:

$$
\begin{gathered}
N_{1}=35 I_{2}, N_{2}=-150 I_{2}, S_{1}=5 I_{2}, \\
\Delta=0.05, \sigma=0.45, \gamma=0.5, \\
\epsilon=0.5, \beta=0.6, B=500 \quad 500 .
\end{gathered}
$$

Figs. 5 and 6 exhibit the trajectory tracking performance of the studied system. For both links, there is a good correspondence between the red solid line representing the controlled position and the green dotted line representing the desired position.

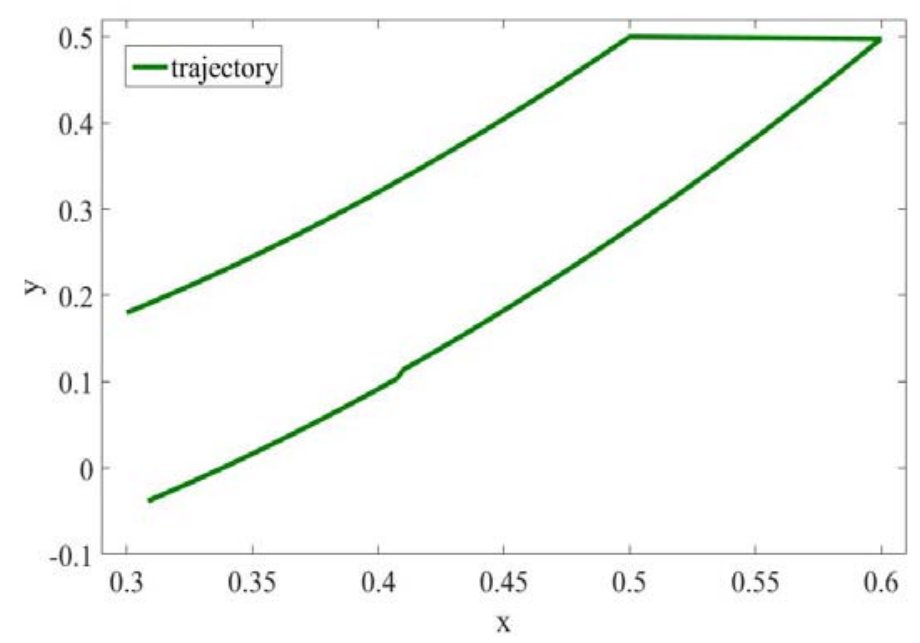

Fig. 4 End-effector trajectory in $x-y$-plane.

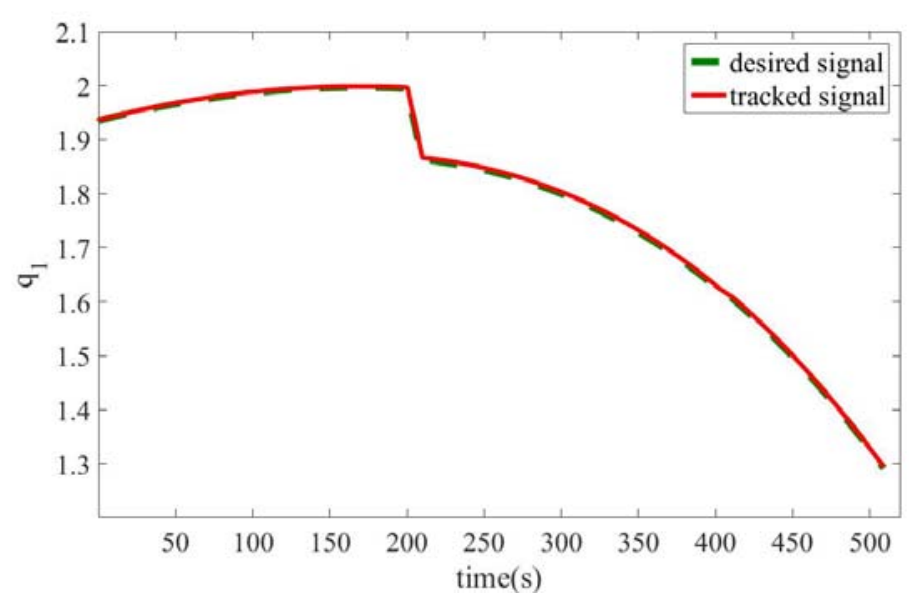

Fig. 5 Position tracking of link 1: $q_{d 1}$ and $q_{1}$. 


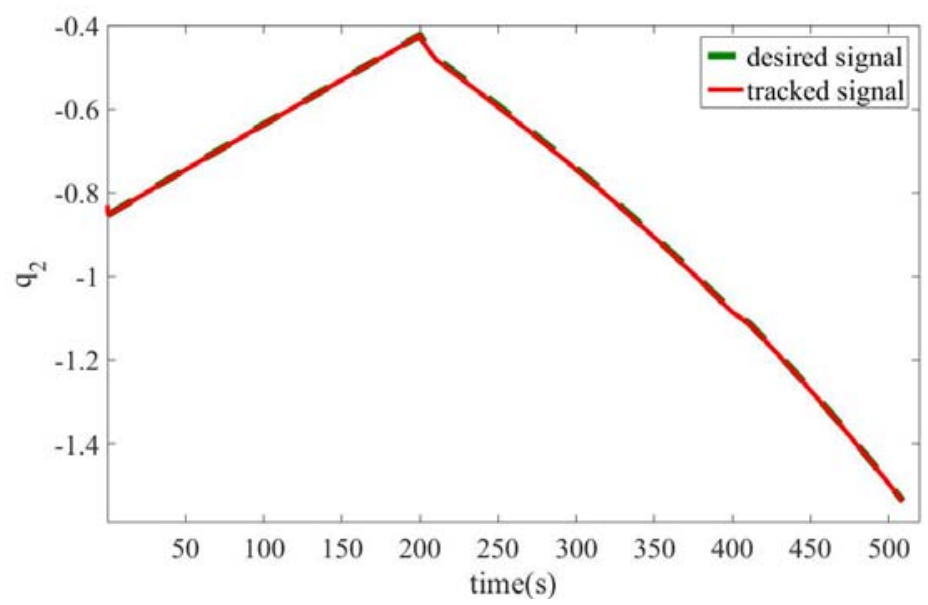

Fig. 6 Position tracking of link 2: $\mathbf{q}_{\mathrm{d} 2}$ and $\mathbf{q}_{2}$.

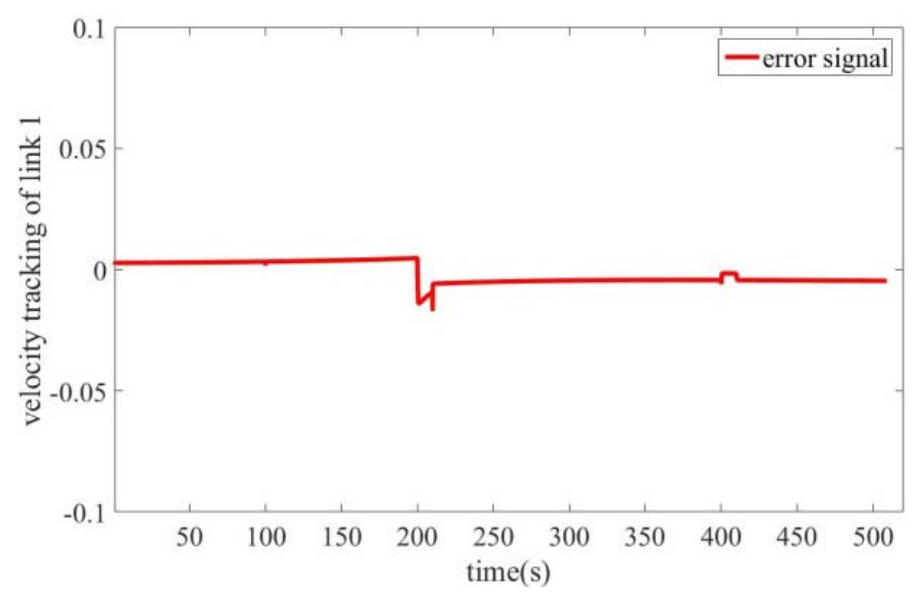

Fig. 7 Velocity error of link 1.

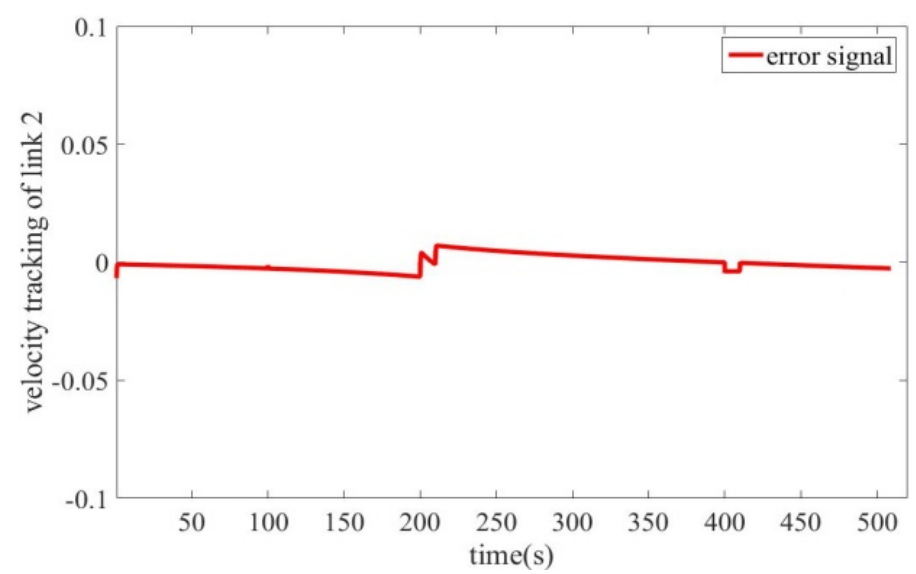

Fig. 8 Velocity error of link 2.

The velocity error, i.e., the difference between the desired and the actual velocity of link 1 and link 2 is shown in Figs. 7 and 8. The error signal's adjacency to 0 verifies the expected tracking performance of the suggested system.
The present simulation verifies the tracking performance of the developed control scheme for the chosen test path with switching constraints and internal as well as external uncertainties. This is a promising first step for future practical implementation in both 
laboratory and industrial environments.

\section{Conclusions}

The control scheme developed in this work combines neurologically-inspired BELBIC and sliding mode control with a conventional control term. The purpose is the compensation of unpredictable changes in internal and external circumstances. The former is considered in the simulation as a 10-percentaged deviation from expected robotic dynamics parameters. The latter is implemented first as a time-dependent external disturbance-function and second as switching constraints due to a discontinuous trajectory to be tracked.

An interplay of inputs relating to information about the surrounding environment through error-signals of position and sliding surfaces and of emotional signals adapting the learning process and regulating the future behaviour based on expected rewards allows the compensation of switching constraints and uncertainties. The expected stable performance is validated through numerical simulations.

The application of the suggested controller is freeform-trajectory tracking of industrial robotic manipulators as found in contact-based manufacturing processes, e.g., in freeform grinding.

\section{Acknowledgement}

This work has been done in the framework of the European Union supported INTERREG GR-project “ROBOTIX-Academy”, i.e., a cross-boarder research cluster for industrial robotics regrouping institutions from Luxembourg, France, Belgium and Germany. (http://robotix.academy/)

\section{References}

[1] Van Cuong, P., and Nan, W. Y. 2015. “Adaptive Trajectory Tracking Neural Network Control with Robust Compensator for Robot Manipulators.” Neural Computing and Applications. DOI 10.1007/s00521-015-1873-4.

[2] Roopaei, M., and Zolghadri Jahromi, M. 2009. "Chattering-free Fuzzy Sliding Mode Control in MIMO Uncertain Systems.” Nonlinear Analysis 71 (10): 4430-7.
[3] Li, T. S., and Huang, Y. 2010. "MIMO Adaptive Fuzzy Terminal Sliding-mode Controller for Robotic Manipulators.” Information Sciences 180 (23): 4641-60.

[4] Li, Y., and Yang, G. 2015. "Robust Fuzzy Adaptive Fault-Tolerant Control for a Class of Nonlinear Systems with Mismatched Uncertainties and Actuator Faults.” Nonlinear Dynamics 81: 395-409.

[5] Liberzon, D. 2003. Switching in Systems and Control. Birkhäuser.

[6] Pagilla, P. R., and Yu, B. 2001. "Adaptive Control of Robotic Surface Finishing Processes.” In Proceedings of the American Control Conference, 630-5.

[7] Yu, L., Fei, S., Huang, J., and Yu, G. 2014. "Trajectory Switching Control of Robotic Manipulators Based on RBF Neural Networks.” Circuits, Systems and Signal Processing 33 (4): 1119-33.

[8] Jasim, I. F., and Plapper, P. W. 2013. “Adaptive Sliding Node Dontrol of Switched Constrained Robotic Manipulators." In Proceedings of 11th IEEE International Conference on Industrial Informatics (INDIN), 305-10.

[9] Tramper, J. J., and Flanders, M. 2013. "Predictive Mechanisms in the Control of Contour Following." Experimental Brain Research 227 (4): 535-46.

[10] Huang, L., Ge, S. S., and Lee, T. H. 2003. "Fuzzy Unidirectional Force Control of Constrained Robotic Manipulators.” Fuzzy Sets and Systems 134: 135-46.

[11] Yin, Y., Hu, H., and Xia, Y. 2004. “Active Tracking of Unknown Surface Using Force Sensing and Control Technique for Robot.” Sensors and Actuators A: Physical 112: 313-9.

[12] Lucas, C., Shahmirzadi, D., and Sheikholeslami, N. 2004. "Introducing BELBIC: Brain Emotional Learning Based Intelligent Controller.” Intelligent Automation and Soft Computing 10 (1): 11-22.

[13] Morén, J., and Balkenius, C. 2000. “A Computational Model of Emotional Learning in the Amygdale." In Proceedings of the 6th International Conference on Simulation of Adaptive Behavior 32.

[14] Balkenius, C., and Morén, J. 2001. "Emotional Learning: A Computational Model of the Amygdala." Cybernetics and Systems 32 (6): 611-36.

[15] Aghaee, S. A., Lucas, C., and Zadeh, K. A. 2012. "Applying Brain Emotional Learning Based Intelligent Controller (Belbic) to Multiple-Area Power Systems.” Asian Journal of Control 14 (6): 1580-8.

[16] Daryabeigi, E., Abjadi, N. R., and Arab Markadeh, G. R. 2014. "Automatic Speed Control of an Asymmetrical Six-phase Induction Motor Using Emotional Controller (BELBIC).” Journal of Intelligent and Fuzzy Systems 26 (4): 1879-92. 
[17] Yi, H. 2015. “A Sliding Mode Control Using Brain Limbic System Control Strategy for a Robotic Manipulator.” International Journal of Advanced Robotic Systems 12 (158).

[18] Liu, J. K., and Wang, X. H. 2011. Advanced Sliding Mode Control for Mechanical Systems: Design, Analysis and MATLAB Simulation. Beijing: Tsinghua University Press; Berlin Heidelberg: Springer-Verlag.

[19] Slotine, J. -J. E., and Li, W. 1991. Applied Nonlinear Control. Upper Saddler River, NJ:Prentice Hall.

[20] Shah, H., and Chakravarty, S. 2009. "Kinematic and Dynamic Control of a Two Link Manipulator.” Control. 